\title{
Using a Density Dependent Population Model to Examine Sperm Whale Population Dynamics in the Gulf of Mexico Before and After an Environmental Disturbance
}

\author{
Mark Dibbs* \\ Advisor: Dr. Amy Veprauskas $\uparrow$
}

\begin{abstract}
In this paper, we develop a density dependent model to describe sperm whale population dynamics in the Gulf of Mexico. For this model, we consider the stability of the extinction equilibrium and prove the existence and uniqueness of a positive equilibrium. We then examine the stability of the positive equilibrium and substantiate the results with numerical simulations using Matlab. Next, we consider the effect of a disturbance, such as the Deepwater Horizon oil spill, on the sperm whale population in the Gulf of Mexico. Describing a disturbance as an event that results in reductions in survival rates for a certain period of time, we examine the recovery time of a sperm whale population following a disturbance, which we define to be the length of time it takes the population to return to a certain percentage of its asymptotic equilibrium value. Through testing various recovery threshold values, reductions in survival rates, and lengths of time over which the survival rates are reduced, we find that the recovery time is sensitive to each of these variables; depending on the values of these three quantities, the recovery time can last anywhere between a few years and many centuries.
\end{abstract}

\section{Introduction}

The sperm whale (Physeter macrocephalus) is a marine mammal whose population is growing at a slow rate, a fact that contributes to the population's relative fragility [6, 21]. Female sperm whales in the Gulf of Mexico (GoM), which are the focus of this study, rarely leave that area over the course of their lives [20]. Thus, in general, sperm whales in the GoM make up a population distinct from sperm whales in any other body of water on the globe. For example, GoM sperm whales tend to be smaller in size and vary genetically from sperm whales in the Atlantic Ocean [11,20]. Since GoM sperm whale populations are relatively fragile and individuals rarely leave the gulf, disturbances, such as oil spills, could have disastrous effects on the population.

The Deepwater Horizon (DWH) oil spill in 2010, which was the largest oil spill to ever occur in United States waters, has had vast negative effects on populations of marine animals in the GoM $[3,13,18]$. Acoustic studies show that sperm whales were present in the areas near the oil spill $[2,16]$. Currently, there is little available information about the effects of the spill on sperm whales in particular, but it is known that oil spills can cause many adverse physiological effects on marine animals. For example, the vapors released from oil are known to cause inflammation to soft tissues, and dispersants in oil can be toxic to sperm whale skin and can even induce genetic abnormalities [9, 22]. For other marine mammals, these toxicants have been shown to substantially decrease vital rates. For example, studies have demonstrated that oil spills can have both lethal and sublethal effects on killer whales and bottlenose dolphins $[12,14]$. While sperm whales are markedly different from bottlenose dolphins and killer whales, it is nonetheless clear that oil spills have the potential to negatively affect the population dynamics of many marine species, including sperm whales [1].

To examine the possible effects of disturbances such as the DWH oil spill on a sperm whale population, we begin by developing and analyzing a density dependent model based on the linear model developed by Chiquet et al. (2013). We introduce density dependence in this model because sperm whale

* Yale University, New Haven, CT 06520. Email: mark.dibbs@yale.edu. Research was conducted while the author was at the Episcopal School of Acadiana, Broussard, LA 70518.

$\dagger$ Department of Mathematics, University of Louisiana-Lafayette, Lafayette, LA 70504. Email: aveprauskas@ louisiana.edu. This paper was presented at the Mathematical Association of America section meeting at the University of Louisiana-Lafayette in March 2018 and won first place in the undergraduate student paper competition. 
populations follow a bottom-up model in which intraspecific competition for resources, not predators or interspecific competition, is a major factor that affects population size [21]. We assume fecundity is dependent on density because density appears to be most regulated by food availability, and there has been an observed link between food availability and reproduction. For instance, after a decade of whaling near the South African coast, pregnancy rates of sperm whales increased by fifteen percent [4].

Following Ackleh et al. (2017), in order to analyze the effects of a disturbance on sperm whale populations, we constantly reduce survival rates over a set amount of time before returning these rates to their original levels [1]. In this paper, we only analyze lethal impacts of environmental disturbances on sperm whales, which is why we only account for reductions in survival rates. However, we could also investigate sublethal impacts by examining the effects of various reductions in fecundity; in such an analysis, we would expect to obtain similar results [1]. We analyze the effects of reductions in survival rates because we assume that a disturbance such as an oil spill decreases the survival probability of sperm whales in each of the five life stages. We then calculate the recovery time, defined as the amount of time it takes for the total population of female sperm whales to reach $95 \%$ of its asymptotic equilibrium value [19]. We also consider other threshold values to analyze how sensitive the recovery time is to the threshold value. While this environment is highly simplified, it can nonetheless capture the overall dynamics of the population in the aftermath of an environmental disturbance such as the DWH oil spill since it takes into account both the length and the intensity of the disturbance [1]. Therefore, the major question we aim to answer in this paper is the following: using a density dependent population model, how long will it take for the total population of sperm whales to recover after a lethal disturbance?

\section{Model Development}

\section{Density Dependent Sperm Whale Model}

The initial goal of this analysis is to determine how a female population of sperm whales varies over time if we account for density dependence in the fecundity rate. We base our model on the linear model developed by Chiquet et al. (2013), which consists of five different stages: calf, immature (juvenile), mature, mother, and post breeding [6]. The densities of these five stages are denoted by $x_{1}, x_{2}, x_{3}, x_{4}$, and $x_{5}$, respectively; these densities do not sum to 1 . The diagram in Figure 1 represents the life cycle of the sperm whales that will be analyzed in this model. All horizontal arrows represent whales transitioning from one life stage to the next. Arrows whose initial and terminal points are in the same stage represent whales remaining in the same life stage. The arrow from the third stage to the first stage represents mature females giving birth. Post-breeding mothers in the fourth stage nurse their calves and are unavailable to mate. After the calves are weaned, the post-breeding females can breed again and are thus reclassified as mature. This transition is represented by the arrow that begins in the fifth stage and returns to the third stage.

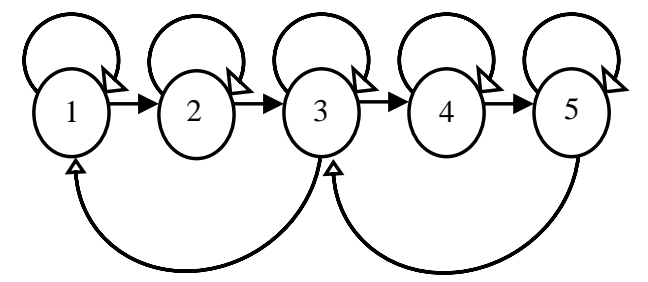

Figure 1. Life cycle of sperm whales used in this model

This life cycle can be represented by the mathematical model

$$
\boldsymbol{x}(t+1)=\boldsymbol{D}(\boldsymbol{x}(t)) \boldsymbol{x}(t)
$$

where $\boldsymbol{x}(t)$ is a column vector that gives the density of whales at each life stage, and $t$ is time in years. The projection matrix $\boldsymbol{D}$ of this model is given by 


$$
\boldsymbol{D}=\left(\begin{array}{ccccc}
P_{1} & 0 & b f\left(x_{2}, x_{3}, x_{4}, x_{5}\right) & 0 & 0 \\
G_{1} & P_{2} & 0 & 0 & 0 \\
0 & G_{2} & P_{3} & 0 & G_{5} \\
0 & 0 & G_{3} & P_{4} & 0 \\
0 & 0 & 0 & G_{4} & P_{5}
\end{array}\right) .
$$

Here, we define $\sigma_{i}$ to be the survival probability of an individual in stage $i$ and $\gamma_{i}$ to be the probability that an individual in stage $i$ will move on to stage $i+1$ for $i=1, \ldots, 4$. Furthermore, we define $P_{i}=$ $\sigma_{i}\left(1-\gamma_{i}\right)$, and $G_{i}=\sigma_{i} \gamma_{i}$, making $P_{i}$ the probability of an individual surviving and staying in stage $i$ and $G_{i}$ the probability of an individual surviving and moving on to stage $i+1$ for $i=1, \ldots, 4$. $G_{5}$ is the probability that an individual will survive and move to stage 3 from stage 5 , and the annual fecundity is given by $b \cdot f(\boldsymbol{x})$ where $b$ is the density independent birth rate and $f(\boldsymbol{x})$ represents a density dependent function. We assume that the calf stage, $x_{1}$, does not impact fecundity since calves are living off their mothers' milk and, therefore, are not competing with others for resources.

Throughout this paper, we assume that the function $f(x)$ satisfies the following three assumptions:

$$
\text { (i) } f(0,0,0,0)=1,(i i) \lim _{\|x\| \rightarrow \infty} f(\boldsymbol{x})=0,(i i i) \frac{\partial}{\partial x_{i}} f(\boldsymbol{x})<0 \text { for } i=2, \ldots, 5 \text {. }
$$

(i) is assumed since, when no animals are present, there is no density dependence and the birth rate is at its maximum possible value $b$. (ii) is assumed since, as the number of sperm whales increases without bound, birth rates will decline since the environment will be unable to support that many whales due to limits in resources such as food and space. Finally, (iii) is assumed because that means $f(\boldsymbol{x})$ (and, by extension, the birth rate) decreases as the total population increases, once again due to limitations in resources. These assumptions are consistent with a Type II functional response in which the predator's intake rate decelerates due to the considerable time used in handling prey, whether that be time spent physically searching for prey, eating the prey, or resting between meals. It is also assumed all competing individuals are comparably efficient in catching prey [10].

\section{Model Analysis}

\section{Extinction Equilibrium}

We first examine the existence and stability of the equilibria of model (1). Model (1) has an extinction equilibrium, which is defined to be the state where no individuals are present, that is $\boldsymbol{E}_{\mathbf{0}}=$ $(0,0,0,0,0)$. We wish to determine when the extinction equilibrium is locally asymptotically stable. The stability of the extinction equilibrium is given by Theorem 1 .

Theorem 1. The extinction equilibrium $\boldsymbol{E}_{\mathbf{0}}=(0,0,0,0,0)$ is locally asymptotically stable if $R_{0}<1$ and is unstable if $R_{0}>1$, where

$$
R_{0}=\frac{G_{1} G_{2}\left(1-P_{4}\right)\left(1-P_{5}\right)}{\left(1-P_{1}\right)\left(1-P_{2}\right)\left[\left(1-P_{3}\right)\left(1-P_{4}\right)\left(1-P_{5}\right)-G_{3} G_{4} G_{5}\right]} .
$$

Proof. The stability of the extinction equilibrium relies on the inherent projection matrix, which is defined as the Jacobian of $\boldsymbol{D}(\boldsymbol{x}(t)) \boldsymbol{x}(t)$ evaluated at the extinction equilibrium. Following these steps, the inherent projection matrix $\boldsymbol{D}_{\mathbf{0}}$ becomes

$$
\boldsymbol{D}_{0}=\left(\begin{array}{ccccc}
P_{1} & 0 & b & 0 & 0 \\
G_{1} & P_{2} & 0 & 0 & 0 \\
0 & G_{2} & P_{3} & 0 & G_{5} \\
0 & 0 & G_{3} & P_{4} & 0 \\
0 & 0 & 0 & G_{4} & P_{5}
\end{array}\right) .
$$


Normally, to determine the stability of the extinction equilibrium, we find the dominant eigenvalue of matrix $\boldsymbol{D}_{\mathbf{0}}$ and determine whether it is less than 1 (stable) or greater than 1 (unstable). However, since we cannot find the eigenvalues of the Jacobian explicitly, we can use another method introduced by Cushing (1998). If the dominant eigenvalue of the Jacobian equals 1 , then the inherent net reproduction number $R_{0}$ also equals $1 . R_{0}$ is defined as the predicted number of offspring each calf will produce over the course of its life in the absence of density dependence. When $R_{0}$ is less than 1 , the extinction equilibrium is stable. When $R_{0}$ is greater than 1, the extinction equilibrium is unstable [7]. $R_{0}$ is equal to the dominant eigenvalue of $\boldsymbol{F}$. $(\boldsymbol{I}-\boldsymbol{T})^{-1}$ where $\boldsymbol{I}$ is the identity matrix, and the transition matrix $\boldsymbol{T}$ and fertility matrix $\boldsymbol{F}$ are defined as

$$
\boldsymbol{T}=\left(\begin{array}{ccccc}
P_{1} & 0 & 0 & 0 & 0 \\
G_{1} & P_{2} & 0 & 0 & 0 \\
0 & G_{2} & P_{3} & 0 & G_{5} \\
0 & 0 & G_{3} & P_{4} & 0 \\
0 & 0 & 0 & G_{4} & P_{5}
\end{array}\right), \boldsymbol{F}=\left(\begin{array}{lllll}
0 & 0 & b & 0 & 0 \\
0 & 0 & 0 & 0 & 0 \\
0 & 0 & 0 & 0 & 0 \\
0 & 0 & 0 & 0 & 0 \\
0 & 0 & 0 & 0 & 0
\end{array}\right) .
$$

It can be shown that $R_{0}=b A$ where

$$
A=\frac{G_{1} G_{2}\left(1-P_{4}\right)\left(1-P_{5}\right)}{\left(1-P_{1}\right)\left(1-P_{2}\right)\left[\left(1-P_{3}\right)\left(1-P_{4}\right)\left(1-P_{5}\right)-G_{3} G_{4} G_{5}\right]}
$$

Thus, the extinction equilibrium is stable if $b A<1$, meaning $b<\frac{1}{A}$, and the extinction equilibrium is unstable otherwise. In addition, we note that the following inequality is necessarily true because $G_{i}=\sigma_{i}-P_{i}<1-P_{i}$ for $i=1, \ldots, 5$ :

$$
\left(1-P_{3}\right)\left(1-P_{4}\right)\left(1-P_{5}\right)-G_{3} G_{4} G_{5}>0
$$

\section{Existence and Uniqueness of a Positive Equilibrium}

We return to the model equations to examine the positive equilibria. We obtain the following result which pertains to the existence of a positive equilibrium.

Theorem 2. A unique positive equilibrium exists when $R_{0}>1$.

Proof. A positive equilibrium must satisfy the following five equilibria equations:

$$
\begin{aligned}
& x_{1}=P_{1} x_{1}+b f(\boldsymbol{x}) x_{3} \\
& x_{2}=G_{1} x_{1}+P_{2} x_{2} \\
& x_{3}=G_{2} x_{2}+P_{3} x_{3}+G_{5} x_{5} \\
& x_{4}=G_{3} x_{3}+P_{4} x_{4} \\
& x_{5}=G_{4} x_{4}+P_{5} x_{5} .
\end{aligned}
$$

We first prove that there is a unique equilibrium when $R_{0}>1$ and $f(\boldsymbol{x})$ satisfies assumptions $(i)$, (ii), and (iii).

By solving equation ( $2 e$ ) for $x_{5}$ and equation $(2 b)$ for $x_{2}$, we can substitute these expressions of $x_{5}$ and $x_{2}$ into equation (2c) to obtain an expression in which $x_{3}=H x_{1}$ where

$$
H=\frac{G_{1} G_{2}\left(1-P_{4}\right)\left(1-P_{5}\right)\left(1-P_{1}\right)}{\left(1-P_{1}\right)\left(1-P_{2}\right)\left[\left(1-P_{3}\right)\left(1-P_{4}\right)\left(1-P_{5}\right)-G_{3} G_{4} G_{5}\right]} \text {. }
$$

We observe that $H=\left(1-P_{1}\right) A$. By substituting our expression for $x_{3}$ into equation $(2 a)$, we obtain the equation $x_{1}=P_{1} x_{1}+b\left(1-P_{1}\right) A f(\boldsymbol{x}) x_{1}$, which simplifies to $\left(1-P_{1}\right)-b\left(1-P_{1}\right) A f(\boldsymbol{x})=0$. We define $h\left(x_{1}\right)$ to be the left side of this equation. We can now determine the positive equilibrium by solving for $h\left(x_{1}\right)=0$. By assumption $(i)$ and the substitution of $R_{0}$ for $b A, h(0)=\left(1-P_{1}\right)\left(1-R_{0}\right)$. Since we assume $R_{0}>1$ and since $P_{1}<1, h(0)<0$. Also, by assumption $(i i), \lim _{x_{1} \rightarrow \infty} h\left(x_{1}\right)=\left(1-P_{1}\right)>0$. Finally, by assumption (iii), $f(\boldsymbol{x})$ is strictly decreasing, so $h\left(x_{1}\right)$ is strictly increasing. Thus, the graph of $h\left(x_{1}\right)$ will begin below the horizontal axis, intersect the axis at a single point, and then increase asymptotically to a fixed 
positive value. Since $h\left(x_{1}\right)$ was set equal to $\left(1-P_{1}\right)-b\left(1-P_{1}\right) A f(\boldsymbol{x})$ with the goal of finding where $h\left(x_{1}\right)$ was equal to 0 , and since $h\left(x_{1}\right)=0$ at only one point, we have shown that there exists a single, unique positive equilibrium for this model when $R_{0}>1$. Note that, by this analysis, we have also shown that no positive equilibrium exists for $R_{0}<1$.

\section{Stability of Positive Equilibrium}

We now examine the stability of the positive equilibrium. The following theorem proves the local stability of the positive equilibrium when $R_{0}$ is greater than but near 1 .

Theorem 3. Assume $\left.\frac{\partial f}{\partial x_{i}}\right|_{\epsilon=0}=-c_{i}$ for $i=2, \ldots, 5$ such that $c_{i} \leq c$ where $c$ is a positive constant. The positive equilibrium is locally asymptotically stable in a neighborhood of $R_{0}$ greater than but near 1.

Proof. Since we cannot explicitly solve for the eigenvalues of the Jacobian evaluated at the positive equilibrium, we approximate the eigenvalues in a neighborhood of when the extinction equilibrium destabilizes and the positive equilibrium emerges, that is $R_{0}=1$. To do this, we take $R_{0}$ to be a bifurcation parameter, meaning we assume that $R_{0}$ is a function of a small parameter $\epsilon$. Specifically, $R_{0}(\epsilon)$ is defined as a small perturbation of $R_{0}$ away from 1; we do this in order to determine population trends for $R_{0}$ greater than and near one. We define $R_{0}(\epsilon)=1+\epsilon$ so that $\epsilon=0$ corresponds to $R_{0}(0)=1$. Similarly, we take $x_{1}, x_{2}, x_{3}$, $x_{4}$, and $x_{5}$ as functions of a small parameter $\epsilon$. For $\epsilon \approx 0$, we use a Taylor series expansion to show that $x_{1}(\epsilon)=x_{1}(0)+x_{1}^{\prime}(0) \epsilon=x_{1}^{\prime}(0) \epsilon$. Similar equations can be found for the other four life stages.

We know that $x_{3}=\left(1-P_{1}\right) A x_{1}$ and that $R_{0}=b A$. By substituting these expressions in for equation (2a) and solving for $x_{1}$, we obtain the equation $0=1+R_{0} f\left(x_{1}\right)$. Next, by substituting in the bifurcation parameters, we obtain the equation $0=1+R_{0}(\epsilon) f\left(x_{1}(\epsilon)\right)=1+(1+\epsilon) \cdot f\left(x_{2}\left(x_{1}(\epsilon)\right), \ldots, x_{5}\left(x_{1}(\epsilon)\right)\right.$. We then implicitly differentiate this equation with respect to $\epsilon$ to obtain the equation $0=f(\epsilon)+(1-\epsilon) \frac{d f}{d \epsilon}$, where, by the chain rule, $\frac{d f}{d \epsilon}=\frac{d x_{1}}{d \epsilon} \cdot \sum_{i=2}^{5}\left[\frac{\partial f}{\partial x_{i}} \cdot \frac{d x_{i}}{d x_{1}}\right]$. We now solve for $\frac{d x_{1}}{d \epsilon}$ evaluated at $\epsilon=0$. Using the fact that $\left.\frac{\partial f}{\partial x_{i}}\right|_{\epsilon=0}=-c_{i}$ for $i=2, \ldots, 5$, we determine that $x_{1}{ }^{\prime}(0)=\frac{1}{B}$, where

$$
B=\frac{G_{1} c_{2}\left[\left(1-P_{3}\right)\left(1-P_{4}\right)\left(1-P_{5}\right)-G_{3} G_{4} G_{5}\right]+G_{1} G_{2}\left[c_{3}\left(1-P_{4}\right)\left(1-P_{5}\right)+G_{3}\left(c_{4}\left(1-P_{5}\right)+c_{5} G_{4}\right)\right]}{\left(1-P_{2}\right)\left[\left(1-P_{3}\right)\left(1-P_{4}\right)\left(1-P_{5}\right)-G_{3} G_{4} G_{5}\right]} .
$$

It is possible to approximate the dominant eigenvalue of the Jacobian evaluated at the positive equilibrium in terms of $\epsilon: \lambda(\epsilon)=\lambda(0)+\lambda^{\prime}(0) \epsilon=1+\lambda^{\prime}(0) \epsilon$. Since $\lambda$ must be less than 1 to ensure the stability of the positive equilibrium, $\lambda^{\prime}(0)$ must be negative to ensure stability for the positive equilibrium. It can be shown that $\lambda^{\prime}(0)=\frac{\boldsymbol{v}(0) \boldsymbol{J}^{\prime}(0) \boldsymbol{w}(0)}{\boldsymbol{v}(0) \boldsymbol{w}(0)}$, where $\boldsymbol{J}$ is the derivative of the Jacobian matrix with respect to $\epsilon$ evaluated at $\epsilon=0$ and $\boldsymbol{v}$ and $\boldsymbol{w}$ are the corresponding left and right eigenvectors, respectively, normalized so that $\boldsymbol{v}(0) \boldsymbol{w}(0)=1$ [7]. Using the fact that $x_{1}{ }^{\prime}(0)=\frac{1}{B}$, this term calculates to

$$
\lambda^{\prime}(0)=\frac{-c_{3}}{A} x_{3}{ }^{\prime}(0)+\frac{H}{A}-\frac{H}{A}\left[c_{2} x_{2}{ }^{\prime}(0)+2 x_{3}{ }^{\prime}(0)+x_{4}{ }^{\prime}(0)+x_{5}{ }^{\prime}(0)\right] .
$$

By solving for the other derivatives, we obtain

$$
\lambda^{\prime}(0)=-\left(1-P_{1}\right) \cdot \frac{c_{2}\left(1-P_{2}\right)\left[\left(1-P_{3}\right)\left(1-P_{4}\right)\left(1-P_{5}\right)-G_{3} G_{4} G_{5}\right]+c_{3} G_{1} G_{2}\left(1-P_{4}\right)\left(1-P_{5}\right)}{c_{2} G_{1}\left[\left(1-P_{3}\right)\left(1-P_{4}\right)\left(1-P_{5}\right)-G_{3} G_{4} G_{5}\right]+G_{1} G_{2}\left[c_{3}\left(1-P_{4}\right)\left(1-P_{5}\right)+G_{3}\left(c_{4}\left(1-P_{5}\right)+c_{5} G_{4}\right)\right.} .
$$

Since this term is negative, the positive equilibrium is stable when $R_{0}$ is greater than but near 1 .

\section{Parameter Values and Numerical Simulations}


Based on information available from the literature, Chiquet et al. estimated the parameters for the linear model, where $f(\boldsymbol{x}) \equiv 1$. These values are given in Table 1 . Again, $\sigma_{i}$ represents the survival probability (in decimal form) of an individual in stage $i$, and $\gamma_{i}$ represents the probability (in decimal form) that an individual in stage $i$ will move on to stage $i+1$ for $i=1, \ldots, 4$. We use these same parameters in our model. We note that since the birth rate $b$ was calculated based on the biologically determined interbirth interval, it is the maximum possible fecundity and does not take into account the effect of density.

Table 1. Vital rates obtained from Chiquet et al. (2013)

\begin{tabular}{|c|c|c|c|c|c|c|c|c|c|c|}
\hline$\sigma_{1}$ & $\sigma_{2}$ & $\sigma_{3}$ & $\sigma_{4}$ & $\sigma_{5}$ & $\gamma_{1}$ & $\gamma_{2}$ & $\gamma_{3}$ & $\gamma_{4}$ & $\gamma_{5}$ & $b$ \\
\hline 0.9070 & 0.9424 & 0.9777 & 0.9777 & 0.9777 & 0.4732 & 0.1151 & 0.2586 & 0.4920 & 0.4920 & 0.1250 \\
\hline
\end{tabular}

For the remainder of this paper, we consider the specific density dependent fecundity function

$$
f(\boldsymbol{x})=\frac{1}{1+c\left(x_{2}+x_{3}+x_{4}+x_{5}\right)} \text {. }
$$

This function assumes a constant value of $c$. We choose this function in particular because this model, which is a modified Beverton-Holt function, has been used extensively in the literature to describe population dynamics of marine mammals such as common bottlenose dolphins, grey seals, and spinner dolphins [19]. However, we note that there are other possible functional forms that satisfy $(i)$, (ii), and (iii), such as $f(\boldsymbol{x})=$ $e^{-c\left(x_{2}+x_{3}+x_{4}+x_{5}\right)}$. To choose $c$ we assume that the positive equilibrium consists of 381 individuals, which was estimated to be the number of female sperm whales in the GoM prior to the DWH oil spill [20]. This assumption results in $c=0.0045$.

In Figure 2, we verify the stability results from the previous sections. For this model, the dominant eigenvalue of the inherent projection matrix, $\lambda=1.0096$, is greater than 1 , meaning that the extinction equilibrium is unstable. In Figure 2 (left), we change $b$ to $b=0.05$ to show that the extinction equilibrium is stable when $R_{0}<1$. In Figure 2 (right), we use the value of $b$ from Chiquet et al. (2013), $b=0.1250$ and the other parameters remain the same as in [6]. This figure shows that the density dependent model brings about stability for positive equilibria when $R_{0}>1$. Through exploring different values of $b$, it appears that the positive equilibrium remains stable for larger values of $R_{0}$ not near 1 . Figure 2 (left) was generated assuming an initial population size of 381 with individuals distributed between the stages according to the stable stage distribution, defined as the right eigenvector associated with the dominant eigenvalue $\lambda$ of the inherent projection matrix $\boldsymbol{D}_{\mathbf{0}}$. This stable stage distribution is

$$
\left(\begin{array}{lllll}
0.0850 & 0.2077 & 0.3617 & 0.1783 & 0.1672
\end{array}\right)^{T},
$$

from Chiquet et al. (2013), where $T$ denotes the transpose of a vector [6]. Since there are a total of 381 female sperm whales in the gulf, the initial population vector of sperm whales prior to the DWH oil spill is given by

$$
\left(\begin{array}{lllll}
32 & 79 & 138 & 68 & 64
\end{array}\right)^{T} \text {. }
$$

Figure 2 (right) was generated with the same stable stage distribution but with an initial population half the size of the one given above in order to demonstrate that the positive equilibrium can be achieved regardless of the initial population size. 

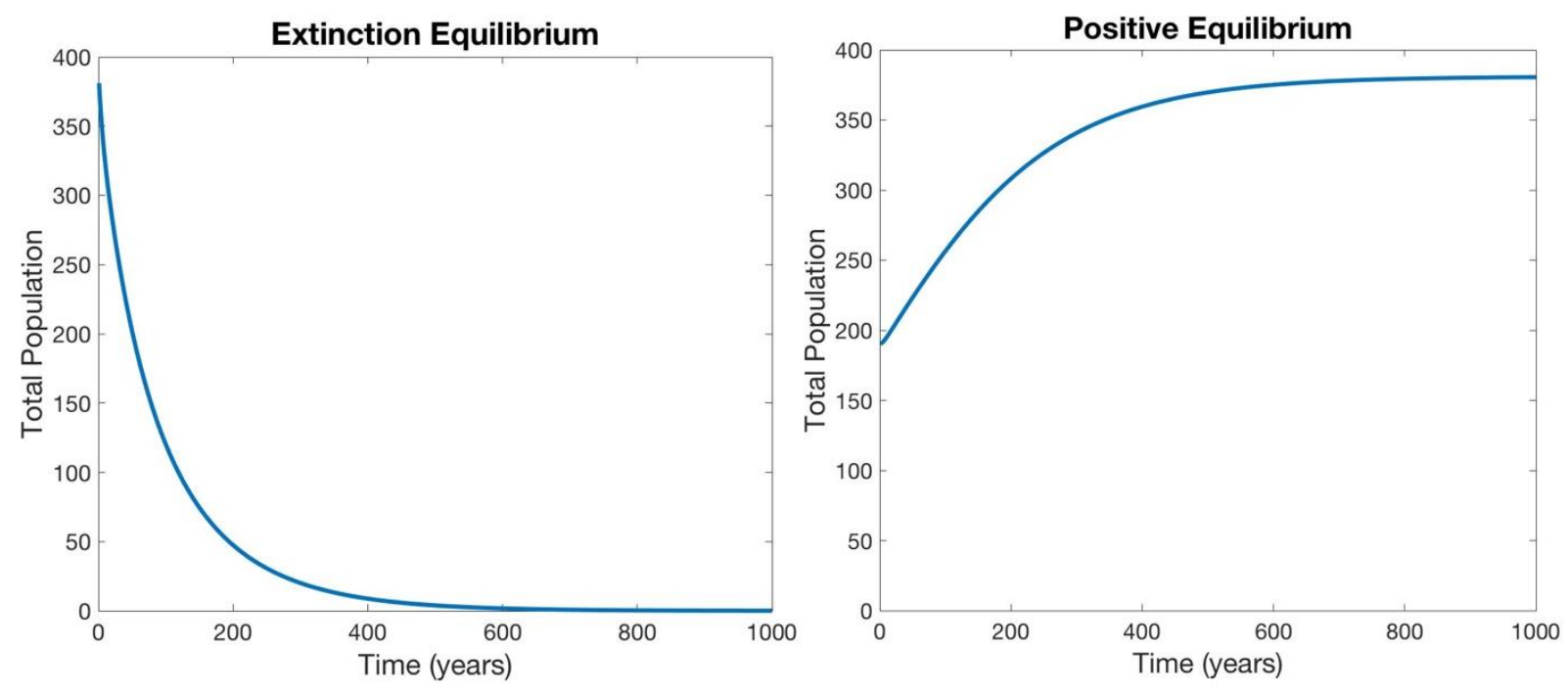

Figure 2. (left) Extinction equilibrium where $b=0.05$; (right) Positive equilibrium where $b=0.125$

\section{Population Recovery Following a Disturbance}

In this section we examine the effects of reductions in survival rates (which simulate the effects of a disturbance, such as the DWH oil spill) on sperm whale population recovery times, defined as the amount of time it takes the population to return to a certain percentage of its original density. To produce the effect of a disturbance, the survival rates $\sigma_{i}$ at each of the life stages will be reduced proportionally by $\epsilon_{0}$ for a certain period of time $T_{c}$ before the survival rates return to their original levels. We incorporate this information into a function, $\hat{\sigma}_{i}\left(\epsilon_{0}(t)\right)$, that will replace $\sigma_{i}$ :

$$
\hat{\sigma}_{i}(t)=\left\{\begin{array}{lc}
\sigma_{i}\left(1-\epsilon_{0}\right), & 0 \leq t<T_{c} \\
\sigma_{i}, & t \geq T_{c}
\end{array}\right.
$$

We assume that 381 is the equilibrium size for the population and, following Schwacke et al. (2017), define the recovery time to be the amount of time it takes for sperm whale numbers to reach $95 \%$ of that value, i.e. 362 individuals $[19,20]$. We assume that the initial population is distributed according to the same equilibrium distribution and initial population size given above.

Using values of $\epsilon_{0}$ from 0.01 to 0.2 and values of $T_{c}$ from 2 to 40 years, we develop the contour plot of recovery time shown in Figure 3 (left). If survival rates are decreased by just $2 \%$ for 10 years, the recovery time is 213 years. A $4 \%$ reduction for just 4 years results in a recovery time of 177 years. Evidently, even small reductions in survival rates or short periods of time over which these reductions take place can have devastating impacts on sperm whale population dynamics. If we instead define the recovery time to be how long it takes for the population to return to $90 \%$ of its original levels, i.e. 343 individuals, we can create a new contour graph, which is shown in Figure 3 (right). The results in this graph show that the effects of a disturbance are more optimistic. A $2 \%$ reduction over 10 years will result in a 101-year recovery period, and a $4 \%$ reduction over 4 years results in a recovery period of 64 years. 

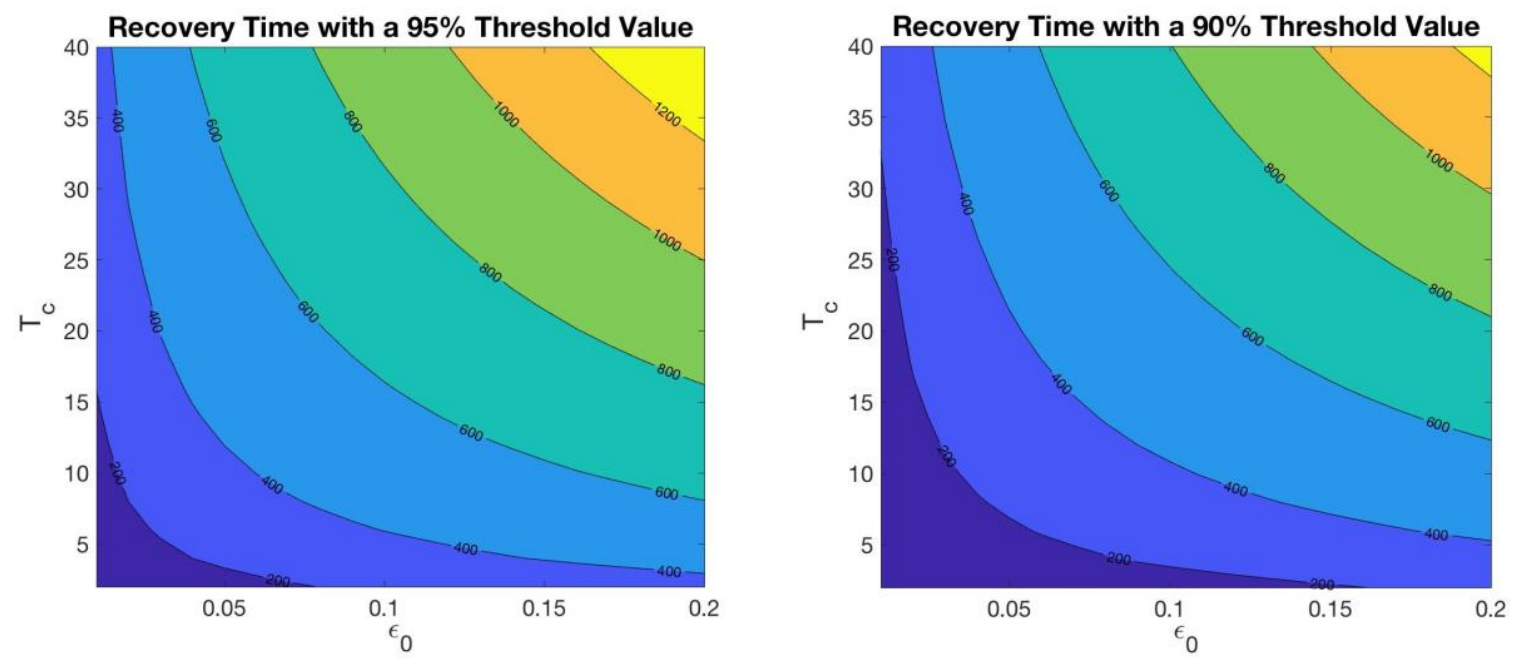

Figure 3. Time needed for the population to return to 95\% (left) or 90\% (right) of its equilibrium value

Since the recovery time appears to be sensitive to the threshold value, we wish to analyze various other threshold values. The plot shown in Figure 4 illustrates the recovery time if we change the threshold value to be between $75 \%$ of the original density (286) and $95 \%$ of the original density (362), using 5\% intervals. We use two different values of $T_{c}$ and three different values of $\epsilon_{0}$ to obtain a wide range of results. As the plot shows, the recovery time increases somewhat linearly as the threshold value increases. In fact, when each of the six curves are fit to a regression line, each of the regression lines has the identical slope with an R-squared value of 0.959. It appears to be the case that regardless of the values of $T_{c}$ and $\epsilon_{0}$, increasing the threshold value by one individual will, on average, increase the recovery time by approximately 3.35 years. In other words, increasing the threshold value by one percent (i.e., 3.81 individuals), on average increases the recovery time by approximately 12.76 years. However, we note that as the threshold value increases, the slope of the regression line also increases. For example, between a $90 \%$ and $95 \%$ threshold value, increasing the threshold value by one individual will increase the recovery time by approximately 22 years. Additionally, as expected, the recovery time increases as the threshold value increases and as the values of $\epsilon_{0}$ or $T_{c}$ increase.

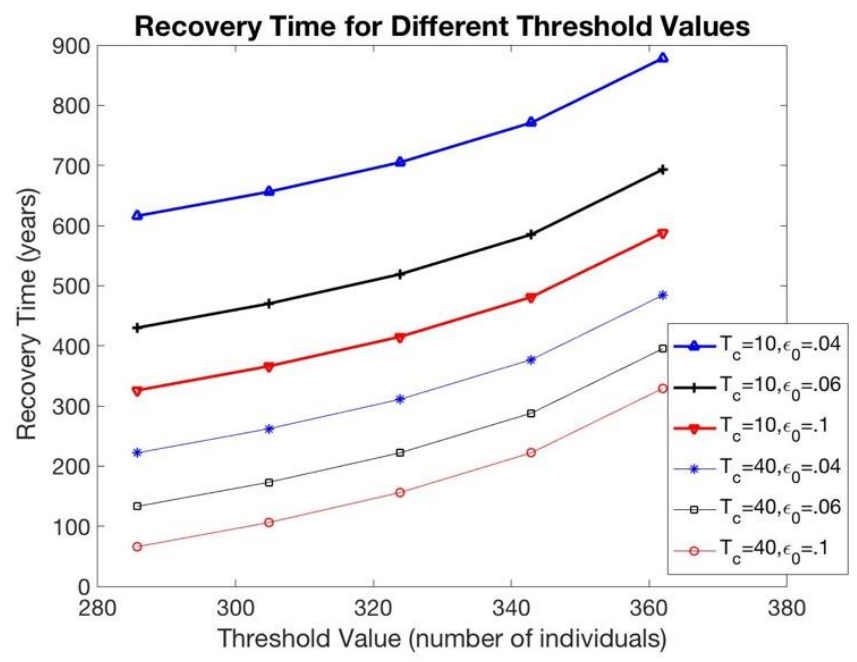

Figure 4. Recovery time for a range of threshold values and different values of $\epsilon_{0}$ and $T_{C}$

\section{Discussion}


Using what little information is known about sperm whale populations in the GoM, studies have estimated that the value for the inherent net reproduction number of sperm whales is indeed greater than 1, but just marginally greater than 1 . This means that the population is relatively fragile and large disturbances, such as oil spills or other forms of chemical or noise pollution, could drive the population to extinction [5]. The Deepwater Horizon oil spill in 2010 had demonstrated negative effects on sperm whale populations. Researchers have found higher levels of DNA-damaging metals found in oil like chromium and nickel in sperm whales in the GoM compared to the rest of the world. This can negatively impact reproduction and development of sperm whales and could even have carcinogenic effects [15]. Another ongoing effect of the spill is that sperm whales are spending less time foraging in ideal locations in the GoM, meaning that these whales may be forced into less suitable areas where increased sperm whale numbers are not sustainable [15].

In this paper, we first developed a density-dependent sperm whale population model as an extension of the linear model developed by Chiquet et al. (2013) in an attempt to more accurately represent sperm whale population dynamics in the GoM. We then analyzed the stability of the extinction equilibrium and we proved the existence of a unique positive equilibrium. We showed that the positive equilibrium is locally asymptotically stable in a neighborhood of $R_{0}$ greater than but near 1 . Based on vital rates given by Chiquet et al. (2013) and using a rational density dependent function, we developed and graphed numerical simulations for our model. Finally, we examined the effects of a disturbance that would reduce survival rates and determined that the recovery time can vary between a few years and a few centuries depending on the proportional reduction in survival rates and the length of time over which the survival rates are reduced, as well as on the threshold value used.

Looking forward, more accurate population models will become increasingly important to determine the effects of human interference or natural disasters on sperm whales in the GoM. According to our model, the recovery time is long for even relatively small disturbances. When we redefined the threshold value to be $90 \%$ of the original population, the recovery time decreased substantially. A biologically relevant and more rigorously defined threshold value for determining recovery time would be helpful in future examinations of environmental disasters. Another way to make this model more realistic would be to gain better knowledge of jumbo squid populations in the GoM, the sperm whale's primary food source, and to incorporate these results into a future model. This would allow us to better understand sperm whale population trends and the effects of human interference and stochastic events on the whales. Since recovery times for sperm whales tend to be long, we could also consider the possibility of additional environmental disturbances. In such a scenario, we could determine the probability of extinction if repeated environmental disasters were to occur.

\section{References}

[1] ACKLEH AS, CHIQUET RA, MA B, TANG T, CASWELL H, VEPRAUSKAS A, SIDOROVSKAIA N. (2017), Analysis of lethal and sublethal impacts of environmental disasters on sperm whales using stochastic modeling. Ecotoxicology, 26: 820.

[2] ACKLEH AS, IOUP GE, IOUP JW, MA B, NEWCOMB JJ, PAL N, SIDOROVSKAIA NA, TIEMANN C (2012), Assessing the deepwater horizon oil spill impact on marine mammal population through acoustics: endangered sperm whales. J Acoust Soc Am 131(3):2306-2314

[3] BARLOW J., SWARTZ SL, EAGLE TC, WADE PR (1995), U.S. marine mammal stock assessments: guidelines for preparation, background, and a summary of the 1995 assessments, NOAA 647 Tech. Memo. NMFS-OPR-6, 73.

[4] BEST, P.B. (1979), Social organization in sperm whales, Physeter microcephalus, in Behavior of marine animals. H.E. Winn and B.L. Olla, eds. New York: Plenum Press.

[5] CHIQUET ET AL. (2015), A matrix population model of beaked whales. Neural, Parallel, and Scientific Computations. 
[6] CHIQUET RA, MA B, ACKLEH AS, PAL N, SIDOROVSKAIA N (2013), Demographic analysis of sperm whales using matrix population models. Ecol Model 248.

[7] CUSHING, J. M. (1998). An introduction to structured population dynamics (Vol. 71). SIAM.

[8] ESTES, J.A. ET AL., EDS (2006), Whales, whaling and ocean ecosystems (University of California Press, Berkeley, CA).

[9] GERACI JR (1990), Physiologic and toxic effects on cetaceans. In: Geraci JR, St. Aubin DJ (eds) Sea mammals and oil. Confronting the risks. Academic Press, Inc., San Diego et al., p 167-198

[10] HOLLING, C. S. (1959), Some characteristics of simple types of predation and parasitism. Canadian Entomologist 91:385-398.

[11] JOCHENS A, BIGGS D, BENOIT-BIRD K, ENGELHAUPT D, GORDON J, HU C, JAQUET N, JOHNSON M, LEBEN R, MATE B, MILLER P, ORTEGA-ORTIZ J, THODE A, TYACK P, WURSIG B (2008), Sperm whale seismic study in the Gulf of Mexico: Synthesis report, U. S. Dept. of the Interior, Minerals Management Service, Gulf of Mexico OCS Region, New Orleans, LA, OCS Study MMS 2008-006, p341

[12] LANE SM, SMITH CR, MITCHELL J, BALMER BC, BARRY KP, MCDONALD T, MORI CS ET AL. (2015), Reproductive outcome and survival of common bottlenose dolphins sampled in Barataria Bay, Louisiana, USA, following the Deepwater Horizon oil spill. Proc R Soc Lond B 282(1818):20151944

[13] LEVY JK, GOPALAKRISHNAN C (2010), Promoting ecological sustain- ability and community resilience in the US Gulf Coast after the 2010 Deepwater Horizon oil spill. J Nat Resourc Policy Res 2 (3):297-315

[14] MATKIN CO, SAULITIS EL, ELLIS GM, OLESIUK P, RICE SD (2008), Ongoing population-level impacts on killer whales Orcinus orca following the Exxon Valdezoil spill in Prince William Sound, Alaska. Mar Ecol Prog Ser 356:269-281

[15] MCCORMICK, LACEY (2015), Five years and counting: gulf wildlife in the aftermath of the Deepwater Horizon disaster. National Wildlife Federation.

[16] MERKENS K, MCDONALD M, BAUMANN-PICKERING S, FRASIER K, WIGGINS S, HILDEBRAND J (2013), Deep-diving cetaceans and the deepwater horizon oil spill. J Acoust Soc Am 134:4176

[17] NORRIS, K. S., AND HARVEY, G. W. (1972), "A theory for the function of the spermaceti organ of the sperm whale," NASA SP-262

[18] RAMSEUR JL (2010), Deepwater Horizon oil spill: the fate of the oil. Congressional Research Service, Library of Congress, Washington, DC

[19] SCHWACKE, L.H., THOMAS, L., WELLS, R.S., MCFEE, W.E., HOHN, A.A., MULLIN, K.D., ZOLMAN, E.S., QUIGLEY, B.M., ROWLES, T.K., SCHWACKE, J.H., (2017), Quantifying injury to common bottlenose dolphins from the Deepwater Horizon oil spill using an age-, sex- and class-structured population model. Endanger. Species Res.

[20] WARING GT, JOSEPHSON E, MAZE-FOLEY K, AND ROSEL PE EDS (2012), Sperm whale (Physeter macrocephalus): Northern Gulf of Mexico Stock, U. S. Atlantic and Gulf of Mexico Marine Mammal Stock Assessments-2012, NOAA Tech Memo NMFS NE, 213, 528.

[21] WHITEHEAD H (2003), Sperm whales: social evolution in the ocean. 742 University of Chicago Press, Chicago

[22] WISE CF, WISE JTF, WISE SS, THOMPSON WD, WISE JR. JP, WISE SR. (2014), Chemical dispersants used in the Gulf of Mexico oil crisis are cytotoxic and genotoxic to sperm whale skin cells. Aquat Toxicol 152:335-340 